\title{
A Study on the Use of Semaphoric Gestures to Support Secondary Task Interactions
}

\author{
Maria Karam, m.c. schraefel \\ School of Electronics and Computer Science \\ University of Southampton \\ Southampton, United Kingdom \\ amrk03r|mc@ecs.soton.ac.uk
}

\begin{abstract}
We present results of a study that considers (a) gestures outside the context of a specific implementation and (b) their use in supporting secondary, rather than primary tasks in a multitasking environment. The results show semaphoric gestures offer significant benefits over function keys in such interactions, and how our findings can be used to extend models of design and evaluation for ubiquitous computing environments that support multitasking.
\end{abstract}

\section{Categories \& Subject Descriptors:}

H.5.2 [Information Interfaces and Presentation]: User Interfaces - evaluation/methodology, prototyping, usercentered design, Input devices and strategies (e.g., mouse, touchscreen

\section{General Terms:}

Experimentation, Human Factors, Design

\section{Keywords:}

Semaphoric Gestures, Secondary Tasks, Notification Systems, Ambient Interactions

\section{INTRODUCTION}

Researchers have suggested that hand-based signaling or sign gestures, referred to as semaphoric gestures, are not natural for interacting with computer systems [7, 8]. A particular challenge has been to assert that this style of gestures are not a viable interaction controller since they can be physically taxing and provide less than the $100 \%$ accuracy of function keys [8]. We present results of a study on the use of gestures in a multitasking environment and as support for secondary, rather than primary, task interactions. This work is particularly concerned with reducing the effects of interruptions in multitasking situations. Our findings show that interaction mode is a significant factor for assessing and evaluating interaction performance with secondary tasks in existing systems and as an element for consideration in the design process.

Copyright is held by the author/owner(s).

CHI 2005, April 2-7, 2005, Portland, Oregon, USA.

ACM 1-59593-002-7/05/0004.

\section{RELATED WORK}

We are investigating semaphoric style hand gestures as described by Quek et al. [7]. While Wexelblat [8] and Quek [7] both claim that semaphoric gestures are not natural for computer interactions and represent only a small part of human communication, we suggest that the "small part" is potentially well matched to secondary interactions. Secondary or background tasks are those which can take place concurrently with the primary task [1]. When the demands of the secondary task cause it to become the user's primary focus, referred to as "forced divided attention" [9], negative performance effects on the primary task can occur [2]. Gestures have been used as an approach for reducing these effects, facilitating "eyes-free" interaction as demonstrated with marking menus, for example [4].

Secondary task interactions are often considered a primary concern within notification systems research where a secondary event such as an instant messenger window may interrupt a primary task such as editing a text document [6]. Research in this area investigates the effects of distraction and recovery caused to a primary task by an interruption, looking at methods for reducing distraction through positioning of a notification and prioritizing interruptions, for example [1,3]. To better understand notification systems, McCrickard proposes three critical parameters to describe a notification system; interruption, response and comprehension [1]. Each is measured as high or low values as set by the system designers to address user goals and requirements. Within this framework, ambient systems, for example, may be set at low interruption, low comprehension and low response requirements. An ambient system, characterized as a form of a stand-alone notification system [5] provides persistent, peripherally available content in an unobtrusive manner to the user. It is within this type of system that we situate our study on gestures to support secondary interactions.

\section{APPROACH}

We noticed a persistent appearance of people working with headphones on within our lab, motivating us to consider music as the background activity for our study. 25 people from our lab were interviewed and given questionnaires. Only one of the 25 people surveyed listened to music as a primary activity; the rest listened while engaged in other 
mental or physical activities such as reading, writing or doing household chores. This prompted us to create an ambient music system, using the criteria for ambient systems mentioned above, as our secondary interaction activity. Results from the interviews and the supplemental questionnaire showed that the most common tasks for interacting with music players as selected by respondents were play, next track, previous track, start and stop.

\section{Determining Background Interactions}

Regarding interaction modes, respondents listened to music most frequently on their computers and used the mouse, media keys or global hot keys to control their players. None used a remote control device, even when one existed. The interviews were also video taped and participants were asked to perform their idea of natural semaphoric gestures to signal the main control functions for a music player. Figure 1 summarizes the results, showing that for all but play functions, most participants had a similar gesture in mind for each of the controls.

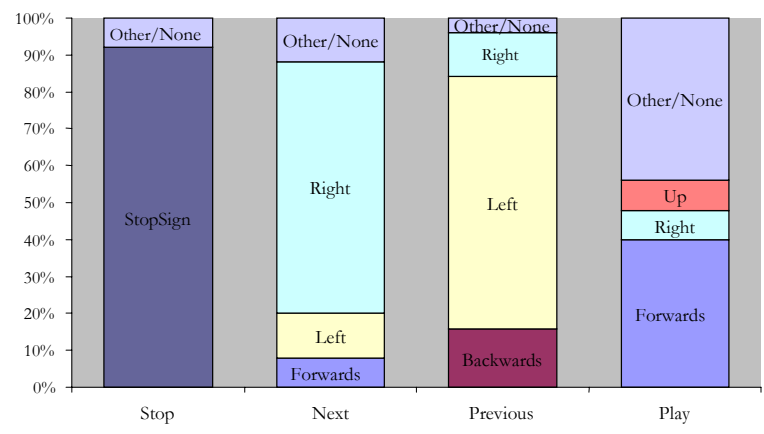

Figure 1. Interviews show users choice of hand gestures.

\section{Determining a Gesture Set}

We developed a set of common gestures based on directional movements performed during the interviews for the stop, play, next and previous track controls on a music player. The gestures consisted of a clockwise circular motion for play based on combining the more commonly observed forwards and right gestures for play during the interviews - a left to right hand wave for next piece (right), right to left hand wave for the previous piece (left) and an open handed halt gesture for stopping playback (stop sign).

\section{STUDY}

To test the hypotheses that semaphoric gestures to control secondary tasks can reduce forced divided attention or distraction from primary tasks in multitasking situations, we designed a multitasking experiment to compare the performance of gestures against standard interaction - in this case, function keys - to manage secondary tasks. Our decision to use a function key interaction as our control condition was motivated by the familiarity and simplicity of function keys for this purpose as shown in our preliminary interviews. A table was set up with a laptop computer running a text editor to be used in the primary task. To the left side was a deck of snap cards with figures and text, and to the right on a sheet of paper, was a wireless computer keyboard used to control the ambient music system. As ambient systems are typically self-contained units, we chose a separate computer, control surface (the wireless keyboard) and speaker system to simulate an ambient system for running the music software. A reference sheet with the task names and corresponding function keys or gestures was provided during the experiment.

Gestures in a ubiquitous system could potentially be recognized from any location in the interaction space, whereas a single physical device, like a remote control, would require the user to spend time locating or manipulating the device to use it. To limit the interaction effects of using different modes, we created a shared interaction zone for both modes. Participants performed gestures and keyboard interactions within a space indicated by the white sheet of paper where the keyboard is situated in Figure 2.

While the typing task was logged on the laptop running the text editor, the control of the music application was partially Wizard of Oz'ed. For the control condition, function keys were mapped directly to Winamp running on a separate computer. A human operator using the wireless keyboard carried out detection of gestures. Participants were instructed to perform a single gesture for each task and then to return to their primary task, so that the reaction time of the system itself was not a factor in the experiment. Mistaken key presses and incorrect gestures were each recorded along with correct input. System errors were not considered in this study as we are investigating gestures as an interaction mode, independent from their implementation.

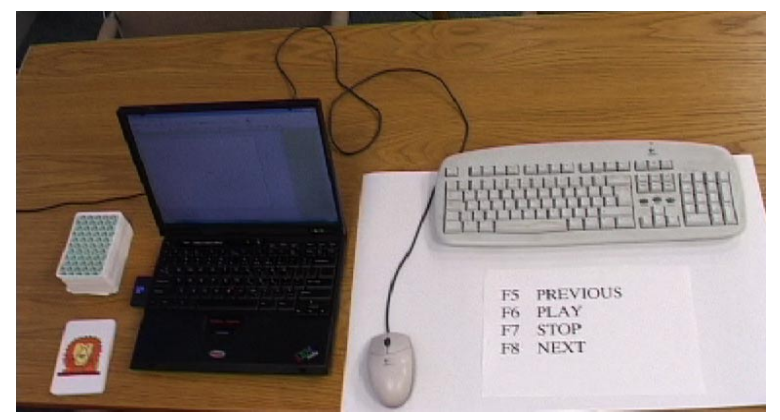

Figure 2. Experiment apparatus set up.

\section{Tasks}

The primary task involved turning over the top card of the deck and typing the name of the image or word displayed on the card into the text editor for the duration of the trials. The secondary task required the user to control the music player using play, stop, next or previous track as indicated by a pop-up notification window that randomly interrupted the primary task, causing a distraction. Participants completed two sets of 20 trials, one using gestures and the other using keyboard. Post experiment interviews had participants compare gestures to the keyboard interaction as equally or more or less satisfying, comfortable, preferred and distracting 
than the keyboard. Participants were given a choice of which hand to use for the experiment and a 10- minute training and practice session before the trials.

Exposure to the treatments was counterbalanced in a withinparticipants, repeated-measures design. The following measures were used to evaluate the effects of gestures on the participants' ability to engage with and complete the secondary task while reducing interruption to the primary task.

\section{Task recovery time}

The time taken for the user to resume interactions with the primary task measured from when the secondary task ends and the primary task resumes.

\section{Secondary task time}

The time taken to complete the secondary task was measured as the time between pressing the space bar on the laptop to indicate the start of the task, and again after the task has been completed. The time taken for the space bar press can be considered a constant factor and should not confound the secondary task times.

\section{Focus loss time}

The total time of distraction from the primary task was measured from the issuing of the alert to the completion of the secondary task.

\section{RESULTS}

We performed analyses comparing mean times for task recovery, secondary task and focus loss times, noting no correlation between dependent variables. We also noticed a gender effect in the task completion time but this was consistent across both the gesture and keyboard modes.

\begin{tabular}{|l|r|r|r|r|r|r|}
\hline & \multicolumn{2}{|c|}{$\begin{array}{c}\text { Task } \\
\text { Completion }\end{array}$} & \multicolumn{2}{c|}{ Task Recovery } & \multicolumn{2}{c|}{ Focus Loss } \\
\hline Mode & Mean & \multicolumn{1}{c|}{ Var. } & Mean & Var. & Mean & \multicolumn{1}{c|}{ Var. } \\
\hline keyboard & 1.81 & .60 & 2.52 & 1.70 & 4.34 & 31.14 \\
gesture & 1.93 & .40 & 2.24 & 1.81 & 3.99 & .81 \\
\hline
\end{tabular}

Figure 3. Summary of results - means and variances of times

User errors in keyboard and gesture commands were also recorded, showing a $.02 \%$ rate for gestures, and a $.03 \%$ rate for function keys. Since there were no correlations between the dependent variables, we ran univariate analysis of variance (ANOVA) for each variable.

\section{Task Completion Time}

A univariate ANOVA was run on the task completion times comparing mode (gesture vs keyboard) against subject, presentation order (gestures or keyboard tested first) and gender. A comparison of mean task completion times showed no significant difference in the time taken for gesture mode over the keyboard. Task completion time also showed no significant effects due to the presentation order of the tasks. Results did show a significant decrease in task completion time on the second trial for both gesture and keyboard interaction, demonstrating a possible learning effect, however these were mode-independent with $\mathrm{F}(1,1)=$ 20.026 at $\mathrm{p}<.001$. Women demonstrated a significantly longer task completion time, independent of mode, with $\mathrm{F}(1,1)=11.003$ at $\mathrm{p}<001$.

\section{Task Recovery Time}

Three univariate ANOVA were run on task recovery time for mode (gesture vs keyboard) and subject, presentation order (gestures or keyboard tested first) and gender. Task recovery time was shown to be significantly lower for the gestures than for the keyboard with $\mathrm{F}(1,1)=2.020$ at $\mathrm{p}<.05$ for all participants and was not affected by order. We did notice a gender effect on the task recovery time with female participants showing significantly lower times than the male participants for both gestures and keyboard with $\mathrm{F}(1,1)=$ 22.324 at $\mathrm{p}<.001$.

\section{Focus Loss Time}

A univariate ANOVA was run on the focus loss time for the mode (gesture vs keyboard) and subject showing no significant differences in the focus loss time with $\mathrm{F}(1,15)=$ 1.339 with $\mathrm{p}=.173$.

\section{Satisfaction}

The post experiment survey results, presented in Figure 4, showed that the gesture mode was preferred overall. Post experiment interviews revealed that all participants would prefer the gesture interaction in a situation where the keyboard was out of reach from their current task as could occur in a ubiquitous computing environment. The results on comfort were split, some reported that the extra movements for gesturing over key presses seemed excessive to some. There is also a level of arm fatigue that con occur with frequent gestures within an extended period of time, however the trade-off in a real-world situation, where gestures would likely be much less frequent than the test condition, was said to be worthwhile.

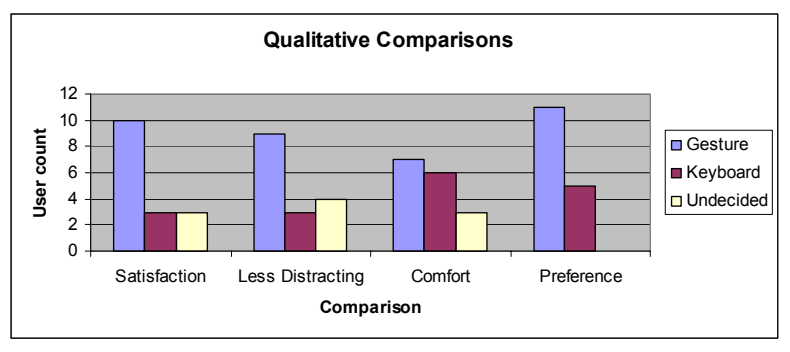

Figure 4. Results from the post experiment questions.

\section{DISCUSSION}

Physical Interaction Requirements and Task Completion No difference in task completion times was found between the gesture and keyboard conditions. Furthermore, in a real ubiquitous computing environment, the interaction zone for a non-gesture based interaction may not always be readily available to the user within reach of their primary task area. 
A remote control may need to be found; a control surface may be at the other end of the room. Thus, the number of steps to complete a simple, one-step gesture press of a button may increase dramatically. Gestures can potentially be detected from anywhere in an environment, thus maintaining a persistent two-step interaction to complete a given secondary task.

\section{Vision requirements and task recovery time}

A more telling result than task completion time is task recovery time. We have shown that task recovery time is significantly shorter when using gestures. Semaphoric gestures can support eyes-free interaction as target acquisition is less demanding for the gestures than for a keyboard. This reduced requirement on the visual channel to complete secondary tasks may account for the significant difference in task recovery times when using gestures as more visual focus remains on the primary task, hence, reducing the threshold for recovering visually-oriented primary task focus.

\section{Gestures: Ease of Use}

Since the number of errors observed for the gesture mode was less than those for keyboard interaction $(0.2 \%$ vs $0.3 \%)$, it appears that, despite the novelty of gestures as an interaction method, gestures were as easy to use accurately as the more familiar keyboard interaction.

\section{CONCLUSION}

While semaphoric gestures have been criticized as an interaction mode over controllers such as function keys, we have shown that there are significant benefits to using gestures for secondary task interactions including reducing task recovery time between completion of the secondary task and returning to the primary task. We also argue that ubiquitous detection of gestures maintains a persistently low number of steps for task interactions, whereas physical device controllers can introduce increased delays in completing the secondary task and potentially degrade in recovery of primary task focus. Our results have also demonstrated that interaction mode is a significant factor for assessing interaction performance with ambient or secondary task systems. We therefore propose that critical parameters used to assess notification systems $[5,6]$ be extended to include interaction mode.

\section{FUTURE WORK}

We plan a follow up empirical Wizard of Oz/technology neutral study to progressively degrade the accuracy of recognition in order to determine the point at which inaccurate detection hits an unacceptable level for secondary task interaction. The results of this study can also be used by designers as quantitative guidelines for minimum semaphoric gesture system performance criteria.

\section{REFERENCES}

1. Chewar, C.M., McCrickard, D.S., Ndiwalana, A., North, C., Pryor, J. and Tessendorf, D., Secondary task display attributes: optimizing visualizations for cognitive task suitability and interference avoidance. in Proceedings of the symposium on Data Visualisation 2002 table of contents, (Barcelona, Spain, 2002), 165 - 171.

2. Czerwinski, M., Horvitz, E. and Wilhite, S., A Diary Study of Task Switching and Interruptions. in Proceedings of the 2004 conference on Human factors in computing systems, (Vienna, Austria, 2004), 175 - 182.

3. Horvitz, E. and Apacible, J., Learning and reasoning about interruption. in Learning and reasoning about interruption, (Vancouver, British Columbia, Canada, 2003), 20 - 27.

4. Kurtenbach, G. and Buxton, W., User learning and performance with marking menus. in Proceedings of the SIGCHI conference on Human factors in computing systems: celebrating interdependence, (Proceedings of the SIGCHI conference on Human factors in computing systems: celebrating interdependence, 1994), 258 - 264.

5. Mankoff, J., Dey, A.K., Hsieh, G., Kientz, J., Lederer, S. and Ames, M., Heuristic evaluation of ambient displays. in Proceedings of the conference on Human factors in computing systems, (Ft. Lauderdale, Florida, USA, 2003), 169 - 176.

6. McCrickard, D.S., Chewar, C.M., Somervell, J.P. and Ndiwalana, A. A model for notification systems evaluation - assessing user goals for multitasking activity. ACM Transactions on Computer-Human Interaction (TOCHI), 10 (4). 312 - 228.

7. Quek, F., McNeill, D., Bryll, R., Duncan, S., Ma, X.-F., Kirbas, C., McCullough, K.E. and Ansari, R. Multimodal human discourse: gesture and speech. ACM Transactions on Computer-Human Interaction (TOCHI), 9 (3). 171 193.

8. Wexelblat, A., Research Challenges in Gestures: Open issues and unsolved problems. in Proceedings of the international gesture workshop on gesture and sign language in human-computer interaction, (1997), 1-11.

9. Wickens, C.D.a.J.G.H. Engineering Psychology and Human Performance. Prentice Hall, 2000, New Jersey, 2000 . 